\title{
Fractional Schrödinger equation
}

\author{
Nick Laskin* \\ University of Toronto \\ IsoTrace Laboratory \\ 60 St. George Street, Toronto, ON, M5S 1A7 \\ Canada
}

\begin{abstract}
Properties of the fractional Schrödinger equation have been studied. We have proven the hermiticity of fractional Hamilton operator and established the parity conservation law for the fractional quantum mechanics. As physical applications of the fractional Schrödinger equation we have found the energy spectrum for a hydrogen-like atom - fractional "Bohr atom" and the energy spectrum of fractional oscillator in the semiclassical approximation. A new equation for the fractional probability current density has been developed and discussed.

We also discuss the relationships between the fractional and the standard Schrödinger equations.

PACS number(s): 03.65.-w, 05.30.-d, 05.40. Fb, 03.65. Db

Keywords: path integral, Lévy motion, fractional differential equation.
\end{abstract}

\section{Introduction}

The Feynman path integral approach to quantum mechanics [1], [2] is in fact integration over Brownian-like quantum mechanical paths. The Brownian motion is a special case of the Lévy $\alpha$-stable random process. In the mid of

*E-mail: nlaskin@rocketmail.com, Fax: 1(416) 9784711 
1930's P. Lévy and A.Y. Khintchine posed the question: When the sum of $N$ independent identically distributed quantities $X=X_{1}+X_{2} \ldots+X_{N}$ has the same probability distribution $p_{N}(X)$ (up to scale factor) as the individual steps $p_{i}\left(X_{i}\right), i=1, \ldots N$ ? The traditional answer is that each $p_{i}\left(X_{i}\right)$ should be a Gaussian, because of a central limit theorem. In other words, a sum of $N$ Gaussians is again a Gaussian. P. Lévy and A.Y. Khintchine proved that there exists the possibility to generalize the central limit theorem [3], [四]. They discovered class of non-Gaussian Lévy $\alpha$-stable (stable under summation) probability distributions. Each $\alpha$-stable probability distribution has a stability index $\alpha$ often called by the Lévy index, $0<\alpha \leq 2$. When $\alpha=2$ the Lévy $\alpha$-stable distribution is transformed to the well-known Gaussian probability distribution or in other words, the Lévy motion is transformed to the Brownian motion [5], [6].

The possibility to develop the path integral over the paths of the Lévy motion was discussed by Kac [7], who pointed out that the Lévy path integral generates the functional measure in the space of left (or right) continues functions having only discontinuities of the first kind.

In the Refs. [8], [9] it was shown that the path integral over the Lévylike quantum-mechanical paths allows to develop the generalization of the quantum mechanics. Namely, if the path integral over Brownian trajectories leads to the well known Schrödinger equation, then the path integral over Lévy trajectories leads to the fractional Schrödinger equation. The fractional Schrödinger equation includes the space derivative of order $\alpha$ instead of the second $(\alpha=2)$ order space derivative in the standard Schrödinger equation. Thus, the fractional Schrödinger equation is the fractional differential equation in accordance with the modern terminology (see, for example, [10- 15]). This is the main point for the term fractional Schrödinger equation or for more general term fractional quantum mechanics, fQM [9]. As it was mentioned above at $\alpha=2$ the Lévy motion becomes the Brownian motion. Thus, the fQM includes the standard QM as a particular Gaussian case at $\alpha=2$. Quantum mechanical path integral over the Lévy paths at $\alpha=2$ becomes the well known Feynman path integral [1], [2].

The paper is organized as follows.

In Sec.2 the quantum mechanical path integral over the Levy paths has been discussed and the 3D fractional Schrödinger equation has been derived in term of the Riesz fractional derivative.

In Sec.3 we prove the hermiticity of fractional Hamilton operator in the 
Hilbert space with scalar product defined by the same way as for conventional quantum mechanics. The parity conservation law for the fractional quantum mechanics has been established.

Time-independent fractional Schrödinger equation has been introduced and its properties have been studied in Sec.4. As physical applications of the time-independent fractional Schrödinger equation we have found (i) the energy spectrum and equation for the orbits radius for a hydrogen-like atom - fractional "Bohr atom", (ii) the energy spectrum of 1D fractional oscillator in the semiclassical approximation.

In Sec.5 a new equation for the fractional probability current density has been derived and discussed.

In the Conclusion we discuss the relationships between the fractional and the well-known Schrödinger equation.

\section{Path integral}

\subsection{Path integral over the Levy paths}

If a particle at an initial time $t_{a}$ starts from the point $\mathbf{r}_{a}$ and goes to a final point $\mathbf{r}_{b}$ at time $t_{b}$, we will say simply that the particle goes from $a$ to $b$ and its path $\mathbf{r}(t)$ will have the property that $\mathbf{r}\left(t_{a}\right)=\mathbf{r}_{a}$ and $\mathbf{r}\left(t_{b}\right)=\mathbf{r}_{b}$. In quantum mechanics, then, we will have an quantum-mechanical amplitude, often called a kernel, to get from the point $a$ to the point $b$. This will be the sum over all of the trajectories that go between that end points and of a contribution from each. If we have the quantum particle moving in the potential $V(\mathbf{r})$ then the fractional quantum-mechanical amplitude $K_{L}\left(\mathbf{r}_{b} t_{b} \mid \mathbf{r}_{a} t_{a}\right)$ may be written as [9]

$$
\begin{gathered}
K_{L}\left(\mathbf{r}_{b} t_{b} \mid \mathbf{r}_{a} t_{a}\right)= \\
\int_{\mathbf{r}\left(t_{a}\right)=\mathbf{r}_{a}}^{\mathbf{r}\left(t_{b}\right)=\mathbf{r}_{b}} \operatorname{Dr}(\tau) \int \operatorname{Dp}(\tau) \exp \left\{\frac{i}{\hbar} \int_{t_{a}}^{t_{b}} d \tau\left[\mathbf{p}(\tau) \dot{\mathbf{r}}(\tau)-H_{\alpha}(\mathbf{p}(\tau), \mathbf{r}(\tau))\right]\right\},
\end{gathered}
$$

where $\hbar$ is the Planck's constant, $\dot{\mathbf{r}}$ denotes the time derivative, $H_{\alpha}(\mathbf{p}(\tau), \mathbf{r}(\tau))$ is the fractional Hamiltonian given by

$$
H_{\alpha}(\mathbf{p}, \mathbf{r})=D_{\alpha}|\mathbf{p}|^{\alpha}+V(\mathbf{r}), \quad 1<\alpha \leq 2,
$$


with the replacement $\mathbf{p} \rightarrow \mathbf{p}(\tau), \mathbf{r} \rightarrow \mathbf{r}(\tau)$ and $\{\mathbf{p}(\tau), \mathbf{r}(\tau)\}$ is the particle trajectory in phase space. The quantity $D_{\alpha}$ has physical dimension

$$
\left[D_{\alpha}\right]=\operatorname{erg}^{1-\alpha} \cdot \mathrm{cm}^{\alpha} \cdot \sec ^{-\alpha}
$$

The phase space path integral $\int_{\mathbf{r}\left(t_{a}\right)=\mathbf{r}_{a}}^{\mathbf{r}\left(t_{b}\right)=\mathbf{r}_{b}} \operatorname{Dr}(\tau) \int \operatorname{Dp}(\tau) \ldots$ in Eq.(11) is defined by

$$
\begin{gathered}
\int_{\mathbf{r}\left(t_{a}\right)=\mathbf{r}_{a}}^{\mathbf{r}\left(t_{b}\right)=\mathbf{r}_{b}} \operatorname{Dr}(\tau) \int \operatorname{Dp}(\tau) \ldots= \\
=\lim _{N \rightarrow \infty} \int_{-\infty}^{\infty} d \mathbf{r}_{1} \ldots d \mathbf{r}_{N-1} \frac{1}{(2 \pi \hbar)^{3 N}} \int_{-\infty}^{\infty} d \mathbf{p}_{1} \ldots d \mathbf{p}_{N} \times
\end{gathered}
$$$$
\exp \left\{i \frac{\mathbf{p}_{1}\left(\mathbf{r}_{1}-\mathbf{r}_{a}\right)}{\hbar}-i \frac{D_{\alpha} \varsigma\left|\mathbf{p}_{1}\right|^{\alpha}}{\hbar}\right\} \times \ldots \times \exp \left\{i \frac{\mathbf{p}_{N}\left(\mathbf{r}_{b}-\mathbf{r}_{N-1}\right)}{\hbar}-i \frac{D_{\alpha} \varsigma\left|\mathbf{p}_{N}\right|^{\alpha}}{\hbar}\right\} \ldots,
$$

here $\varsigma=\left(t_{b}-t_{a}\right) / N$.

The exponential in Eq.(1) can be written as $\exp \left\{\frac{i}{\hbar} S_{\alpha}(\mathbf{p}, \mathbf{r})\right\}$ if we introduce classical mechanical action for the trajectory $\{\mathbf{p}(t), \mathbf{r}(t)\}$ in phase space

$$
S_{\alpha}(\mathbf{p}, \mathbf{r})=\int_{t_{a}}^{t_{b}} d \tau\left(\mathbf{p}(\tau) \dot{\mathbf{r}}(\tau)-H_{\alpha}(\mathbf{p}(\tau), \mathbf{r}(\tau), \tau) .\right.
$$

When $\alpha=2, D_{\alpha}=1 / 2 m,(m$ is the mass of a particle) Eq.(2) is transformed into the well known Hamiltonian with the kinetic energy $\mathbf{p}^{2} / 2 m$ and Eq.(1) becomes the definition of the Feymnam path integral in the phase space representation, see for example [16].

\subsection{Fractional Schrödinger equation}

The kernel $K_{L}\left(\mathbf{r}_{b} t_{b} \mid \mathbf{r}_{a} t_{a}\right)$ which is defined by Eq.(1), describes the evolution of the quantum-mechanical system 


$$
\psi_{f}\left(\mathbf{r}_{b}, t_{b}\right)=\int d \mathbf{r}_{a} K_{L}\left(\mathbf{r}_{b} t_{b} \mid \mathbf{r}_{a} t_{a}\right) \cdot \psi_{i}\left(\mathbf{r}_{a}, t_{a}\right),
$$

where $\psi_{i}\left(\mathbf{r}_{a}, t_{a}\right)$ is the wave function of initial (at the $t=t_{a}$ ) state and $\psi_{f}\left(\mathbf{r}_{b}, t_{b}\right)$ is the wave function of final (at the $t=t_{b}$ ) state.

In order to obtain the differential equation for the wave function $\psi(\mathbf{r}, t)$ we apply Eq.(5) in the special case that the time differs only by an infinitesimal interval $\varepsilon$ from $t_{a}$

$$
\psi(\mathbf{r}, t+\varepsilon)=\int d \mathbf{r}^{\prime} K_{L}\left(\mathbf{r}, t+\varepsilon \mid \mathbf{r}^{\prime}, t\right) \cdot \psi\left(\mathbf{r}^{\prime}, t\right) .
$$

Using Feynman's approximation $\int_{t}^{t+\varepsilon} d \tau V(\mathbf{r}(\tau), \tau) \simeq \varepsilon V\left(\frac{\mathbf{r}+\mathbf{r}^{\prime}}{2}, t\right)$ and the definition given by Eq.(1) we have

$$
\begin{gathered}
\psi(\mathbf{r}, t+\varepsilon)= \\
\int d \mathbf{r}^{\prime} \frac{1}{(2 \pi \hbar)^{3}} \int_{-\infty}^{\infty} d \mathbf{p} \exp \left\{i \frac{\mathbf{p}\left(\mathbf{r}^{\prime}-\mathbf{r}\right)}{\hbar}-i \frac{D_{\alpha} \varepsilon|\mathbf{p}|^{\alpha}}{\hbar}-\frac{i}{\hbar} \varepsilon V\left(\frac{\mathbf{r}+\mathbf{r}^{\prime}}{2}, t\right)\right\} \cdot \psi\left(\mathbf{r}^{\prime}, t\right) .
\end{gathered}
$$

We may expand the left-hand and the right-hand sides in power series

$$
\begin{aligned}
\psi(\mathbf{r}, t)+\varepsilon \frac{\partial \psi(\mathbf{r}, t)}{\partial t}= & \int d \mathbf{r}^{\prime} \frac{1}{(2 \pi \hbar)^{3}} \int_{-\infty}^{\infty} d \mathbf{p} \exp \left\{i \frac{\mathbf{p}\left(\mathbf{r}^{\prime}-\mathbf{r}\right)}{\hbar}\right\} \cdot\left(1-i \frac{D_{\alpha} \varepsilon|\mathbf{p}|^{\alpha}}{\hbar}\right) \times \\
& \left(1-\frac{i}{\hbar} \varepsilon V\left(\frac{\mathbf{r}+\mathbf{r}^{\prime}}{2}, t\right)\right) \cdot \psi\left(\mathbf{r}^{\prime}, t\right) .
\end{aligned}
$$

Then, taking into account the definitions of the Fourier transforms

$$
\psi(\mathbf{r}, t)=\frac{1}{(2 \pi \hbar)^{3}} \int d \mathbf{p} e^{i \frac{p x}{\hbar}} \varphi(\mathbf{p}, t), \quad \varphi(\mathbf{p}, t)=\int d \mathbf{p} e^{-i \frac{\mathbf{p x}}{\hbar}} \psi(\mathbf{r}, t),
$$

and introducing the 3D quantum Riesz fractional derivative' $\left(-\hbar^{2} \Delta\right)^{\alpha / 2}$

\footnotetext{
${ }^{1}$ The Riesz fractional derivative was originally introduced in 17
} 


$$
\left(-\hbar^{2} \Delta\right)^{\alpha / 2} \psi(\mathbf{r}, t)=\frac{1}{(2 \pi \hbar)^{3}} \int d^{3} p e^{i \frac{\mathbf{p r}}{\hbar}}|\mathbf{p}|^{\alpha} \varphi(\mathbf{p}, t),
$$

(here $\Delta=\partial^{2} / \partial \mathbf{r}^{2}$ is the Laplacian) we obtain from Eq.(6)

$\psi(\mathbf{r}, t)+\varepsilon \frac{\partial \psi(\mathbf{r}, t)}{\partial t}=\psi(\mathbf{r}, t)-i \frac{D_{\alpha} \varepsilon}{\hbar}\left(-\hbar^{2} \Delta\right)^{\alpha / 2} \psi(\mathbf{r}, t)-\frac{i}{\hbar} \varepsilon V(\mathbf{r}, t) \psi(\mathbf{r}, t)$.

This will be true to order $\varepsilon$ if $\psi(\mathbf{r}, t)$ satisfies the differential equation

$$
i \hbar \frac{\partial \psi(\mathbf{r}, t)}{\partial t}=D_{\alpha}\left(-\hbar^{2} \Delta\right)^{\alpha / 2} \psi(\mathbf{r}, t)+V(\mathbf{r}, t) \psi(\mathbf{r}, t) .
$$

This is the fractional Schrödinger equation. The space derivative in this equation is of fractional (noninteger) order $\alpha$.

The above consideration is in fact the generalization of the well known Feynman approach to reduce the path integral to the differential equation [园], [2].

Equation (8) may be rewritten in the operator form, namely

$$
i \hbar \frac{\partial \psi}{\partial t}=H_{\alpha} \psi
$$

where $H_{\alpha}$ is the fractional Hamiltonian operator

$$
H_{\alpha}=D_{\alpha}\left(-\hbar^{2} \Delta\right)^{\alpha / 2}+V(\mathbf{r}, t) .
$$

By definition (10) and introducing the momentum operator $\mathbf{p}=i \hbar \boldsymbol{\nabla}$ one may obtain the fractional Hamiltonian $H_{\alpha}$ in the form given by Eq.(2).

Since the kernel $K_{L}\left(\mathbf{r}_{b} t_{b} \mid \mathbf{r}_{a} t_{a}\right)$ thought of as a function of variables $\mathbf{r}_{b}, t_{b}$, is a special wave function (namely, that for a particle which starts at $\mathbf{r}_{a}, t_{a}$ ), we see that $K_{L}$ must also satisfy a fractional Schrödinger equation. Thus for the quantum system described by the fractional Hamiltonian Eq.(10) we have

$$
i \hbar \frac{\partial}{\partial t_{b}} K_{L}\left(\mathbf{r}_{b} t_{b} \mid \mathbf{r}_{a} t_{a}\right)=D_{\alpha}\left(-\hbar^{2} \Delta_{b}\right)^{\alpha / 2} K_{L}\left(\mathbf{r}_{b} t_{b} \mid \mathbf{r}_{a} t_{a}\right)+V\left(\mathbf{r}_{b}, t\right) K_{L}\left(\mathbf{r}_{b} t_{b} \mid \mathbf{r}_{a} t_{a}\right)
$$

where $t_{b}>t_{a}$, and the low index "b" at the $\Delta_{b}$ means that the fractional derivative acts on the variable $\mathbf{r}_{b}$. 


\section{Quantum Riesz fractional derivative}

\subsection{Hermiticity of the fractional Hamilton operator}

The fractional Hamiltonian $H_{\alpha}$ given by Eq.(10) is the Hermitian operator in the space with scalar product

$$
(\phi, \chi)=\int_{-\infty}^{\infty} d \mathbf{r} \phi^{*}(\mathbf{r}, t) \chi(\mathbf{r}, t)
$$

where the $\operatorname{sign} *$ means as usual complex conjugate.

To prove hermiticity of the fractional Hamilton $H_{\alpha}$ let us note that in accordance with definition of the quantum Riesz fractional derivative given by Eq.(17) there exists the integration by parts formula

$$
\left(\phi,\left(-\hbar^{2} \Delta\right)^{\alpha / 2} \chi\right)=\left(\left(-\hbar^{2} \Delta\right)^{\alpha / 2} \phi, \chi\right) .
$$

The average energy of fractional quantum system with Hamiltonian $H_{\alpha}$ is

$$
E_{\alpha}=\int_{-\infty}^{\infty} d \mathbf{r} \psi^{*}(\mathbf{r}, t) H_{\alpha} \psi(\mathbf{r}, t)
$$

Taking into account Eq.(13) we have

$$
E_{\alpha}=\int_{-\infty}^{\infty} d \mathbf{r} \psi^{*}(\mathbf{r}, t) H_{\alpha} \psi(\mathbf{r}, t)=\int_{-\infty}^{\infty} d \mathbf{r}\left(H_{\alpha}^{+} \psi(\mathbf{r}, t)\right)^{*} \psi(\mathbf{r}, t)=E_{\alpha}^{*} .
$$

and as a physical consequence, the energy of a system is real. Thus, the fractional Hamiltonian $H_{\alpha}$ defined by Eq.(10) is the Hermitian or self-adjoint operator in the space with the scalar product defined by Eq.(12)

$$
\left(H_{\alpha}^{+} \phi, \chi\right)=\left(\phi, H_{\alpha} \chi\right)
$$

\subsection{Parity conservation law for the fractional quantum mechanics}

It follows from the definition (7) of the quantum Riesz fractional derivative that 


$$
\left(-\hbar^{2} \Delta\right)^{\alpha / 2} \exp \left\{i \frac{\mathbf{p x}}{\hbar}\right\}=|\mathbf{p}|^{\alpha} \exp \left\{i \frac{\mathbf{p x}}{\hbar}\right\} .
$$

Thus, the function $\exp \{i \mathbf{p x} / \hbar\}$ is the eigenfunction of the $3 \mathrm{D}$ quantum Riesz fractional operator $\left(-\hbar^{2} \Delta\right)^{\alpha / 2}$ with eigenvalue $|\mathbf{p}|^{\alpha}$.

The operator $\left(-\hbar^{2} \Delta\right)^{\alpha / 2}$ is symmetrized fractional derivative, that is

$$
\left(-\hbar^{2} \Delta_{\mathbf{r}}\right)^{\alpha / 2} \ldots=\left(-\hbar^{2} \Delta_{-\mathbf{r}}\right)^{\alpha / 2} \cdots
$$

Because of the property (17) the fractional Hamiltonian $H_{\alpha}$ given by Eq.(10) remains invariant under inversion transformation. Inversion, or to be precise, spatial inversion consists in the simultaneous change in sign of all three spatial coordinates

$$
\mathbf{r} \rightarrow-\mathbf{r}, \quad x \rightarrow-x, \quad y \rightarrow-y, \quad z \rightarrow-z .
$$

Let us denote the inversion operator by $\widehat{P}$. The inverse symmetry is the fact that $\widehat{P}$ and the fractional Hamiltonian $H_{\alpha}$ commute,

$$
\widehat{P} H_{\alpha}=H_{\alpha} \widehat{P}
$$

We can divide the wave functions of quantum mechanical states with a well-defined eigenvalue of the operator $\widehat{P}$ into two classes; (i) functions which are not changed when acted upon by the inversion operator,

$$
\widehat{P} \psi_{+}(\mathbf{r})=\psi_{+}(\mathbf{r})
$$

the corresponding states are called even states; (ii) functions which change sign under the action of the inversion operator,

$$
\widehat{P} \psi_{-}(\mathbf{r})=-\psi_{-}(\mathbf{r})
$$

the corresponding states are called odd states.

Equation (19) express the "parity conservation law"; if the state of a closed fractional quantum mechanical system has a given parity (i.e. if it is even, or odd), then this parity is conserved. 


\section{Time-independent fractional Schrödinger equa- tion}

The special case when the Hamiltonian $H_{\alpha}$ does not depend explicitly on the time is of great importance for physical applications. It is easily to see that in this case there exist the special solution of the fractional Schrödinger equation (8) of the form

$$
\psi(\mathbf{r}, t)=e^{-(i / \hbar) E t} \phi(\mathbf{r}),
$$

where $\phi(\mathbf{r})$ satisfies

$$
H_{\alpha} \phi(\mathbf{r})=E \phi(\mathbf{r})
$$

or

$$
D_{\alpha}\left(-\hbar^{2} \Delta\right)^{\alpha / 2} \phi(\mathbf{r})+V(\mathbf{r}) \phi(\mathbf{r})=E \phi(\mathbf{r}), \quad 1<\alpha \leq 2 .
$$

The equation (22) we call by the time-independent (or stationary) fractional Schrödinger equation.

\subsection{Fractional Bohr atom}

The hydrogenlike potential energy $V(\mathbf{r})$ is

$$
V(\mathbf{r})=-\frac{Z e^{2}}{|\mathbf{r}|} .
$$

Then the fractional Schrödinger equation (22) has a form

$$
D_{\alpha}\left(-\hbar^{2} \Delta\right)^{\alpha / 2} \phi(\mathbf{r})-\frac{Z e^{2}}{|\mathbf{r}|} \phi(\mathbf{r})=E \phi(\mathbf{r}),
$$

and can be treated as fractional eigenvalue problem.

The total energy of considered quantum mechanical system is

$$
E=E_{k i n}+V,
$$

where $E_{k i n}$ is the kinetic energy 


$$
E_{k i n}=D_{\alpha}|\mathbf{p}|^{\alpha}
$$

and $V$ is the potential energy

$$
V=-\frac{Z e^{2}}{|\mathbf{r}|}
$$

It is well known that if the potential energy is a homogeneous function of the co-ordinates and the motion takes place in a finite region of space, there exists a simple relation between the time average values of the kinetic and potential energies, known as the virial theorem (see, page 23, 18]). It follows from the virial theorem that between average kinetic energy (24) and average potential energy (25) there exist the relation

$$
\alpha \bar{E}_{k i n}=-\bar{V},
$$

where the average value $\bar{f}$ of any function of time is defined as

$$
\bar{f}=\lim _{T \rightarrow \infty} \frac{1}{T} \int_{0}^{\infty} d t f(t) .
$$

In order to evaluate the energy spectrum of the fractional hydrogenlike atom let us remind the Niels Bohr postulates [19]:

1. The electron moves in orbits restricted by the requirement that the angular momentum be an integral multiple of $\hbar$, that is, for circular orbits of radius $a_{n}$, the electron momentum is restricted by

$$
p a_{n}=n \hbar, \quad(n=1,2,3, \ldots),
$$

and furthermore the electrons in these orbits do not radiate in spite of their acceleration. They were said to be in stationary states.

2. Electrons can make discontinuous transitions from one allowed orbit corresponding to $n=n_{2}$ to another corresponding to $n=n_{1}$, and the change in energy will appear as radiation with frequency

$$
\omega=\frac{E_{n_{2}}-E_{n_{1}}}{\hbar}, \quad\left(n_{2}>n_{1}\right) .
$$

An atom may absorb radiation by having its electrons make a transition to a higher energy orbit. 
Using the first Bohr's postulate and Eq.(26) yields

$$
\alpha D_{\alpha}\left(\frac{n \hbar}{a_{n}}\right)^{\alpha}=\frac{Z e^{2}}{a_{n}},
$$

from which it follows the equation for the radius of the fractional Bohr orbits

$$
a_{n}=a_{0} n^{\frac{\alpha}{\alpha-1}},
$$

here $a_{0}$ is the fractional Bohr radius (the radius of the lowest, $n=1$ Bohr orbit) defined as,

$$
a_{0}=\left(\frac{\alpha D_{\alpha} \hbar^{\alpha}}{Z e^{2}}\right)^{\frac{1}{\alpha-1}} .
$$

By using Eq.(26) we find for the total average energy $\bar{E}$

$$
\bar{E}=(1-\alpha) \bar{E}_{k i n}
$$

Thus, for the energy levels of the fractional hydrogen-like atom we have

$$
E_{n}=-(\alpha-1) E_{0} n^{-\frac{\alpha}{\alpha-1}}, \quad 1<\alpha \leq 2,
$$

where $E_{0}$ is the binding energy of the electron in the lowest Bohr orbit, that is, the energy required to put it in a state with $E=0$ corresponding to $n=\infty$,

$$
E_{0}=\left(\frac{\left(Z e^{2}\right)^{\alpha}}{\alpha^{\alpha} D_{\alpha} \hbar^{\alpha}}\right)^{\frac{1}{\alpha-1}}
$$

The energy $(\alpha-1) E_{0}$ can be considered as generalization of the Rydberg constant of the standard quantum mechanics. It is easy to see that at $\alpha=2$ the energy $(\alpha-1) E_{0}$ is transformed into the well known expression for the Rydberg constant, Ry $=m e^{4} / 2 \hbar^{2}$.

The frequency of the radiation $\omega$ associated with the transition, say, for example from $k$ to $n, k \rightarrow n$, is,

$$
\omega=\frac{(\alpha-1) E_{0}}{\hbar} \cdot\left[\frac{1}{n^{\frac{\alpha}{\alpha-1}}}-\frac{1}{k^{\frac{\alpha}{\alpha-1}}}\right], \quad(k>n)
$$


The new equations (29)-(33) give generalization of the "Bohr atom" theory. In a special Gaussian case, $\alpha=2$ (standard quantum mechanics) Eqs.(29)-(33) reproduce the well known results of the Bohr theory [19], [20]. The existence of Eqs.(29)-(33) is a result of deviation of fractal dimension $\mathrm{d}_{\text {fractal }}^{(L e ́ v y)}$ of the Lévy-like quantum mechanical path from $2, \mathrm{~d}_{\text {fractal }}^{(L e ́)}=\alpha<2$.

\subsection{Spectrum of the 1D fractional oscillator in semi- classical approximation}

Fractional oscillator introduced in $[8$ is the model with the fractional Hamiltonian operator $H_{\alpha, \beta}$,

$$
H_{\alpha, \beta}=D_{\alpha}\left(-\hbar^{2} \Delta\right)^{\alpha / 2}+q^{2}|\mathbf{r}|^{\beta}, \quad 1<\alpha \leq 2, \quad 1<\beta \leq 2,
$$

where $\mathbf{r}$ is the $3 \mathrm{D}$ vector, $\Delta=\partial^{2} / \partial \mathbf{r}^{2}$ is the Laplacian, the operator $\left(-\hbar^{2} \Delta\right)^{\alpha / 2}$ is defined by the Eq.(7) and $q$ is a constant with physical dimension $[q]=\operatorname{erg}^{1 / 2} \cdot \mathrm{cm}^{-\beta / 2}$.

The 1D fractional oscillator with the Hamilton function $H_{\alpha, \beta}=D_{\alpha}|p|^{\alpha}+$ $q^{2}|x|^{\beta}$ poses an interesting problem for semiclassical treatment. We set the total energy equal to $E$, so that

$$
E=D_{\alpha}|p|^{\alpha}+q^{2}|x|^{\beta},
$$

whence

$$
|p|=\left(\frac{1}{D_{\alpha}}\left(E-q^{2}|x|^{\beta}\right)\right)^{1 / \alpha} .
$$

At the turning points $p=0$. Thus, classical motion is possible in the range $|x| \leq\left(E / q^{2}\right)^{1 / \beta}$.

A routine use of the Bohr-Sommerfeld quantization rule [22] yields

$$
2 \pi \hbar\left(n+\frac{1}{2}\right)=\oint p d x=4 \int_{0}^{x_{m}} p d x=\frac{4}{D_{\alpha}^{1 / \alpha}} \int_{0}^{x_{m}}\left(E-q^{2}|x|^{\beta}\right)^{1 / \alpha} d x,
$$

where the notation $\oint$ means the integral over one complete period of the classical motion, $x_{m}=\left(E / q^{2}\right)^{1 / \beta}$ is the turning point of classical motion. To 
evaluate the integral in the right hand of Eq.(36) we introduce a new variable $y=x\left(E / q^{2}\right)^{-1 / \beta}$. Then we have

$$
\int_{0}^{x_{m}}\left(E-q^{2}|x|^{\beta}\right)^{1 / \alpha} d x=\frac{1}{q^{2 / \beta}} E^{\frac{1}{\alpha}+\frac{1}{\beta}} \int_{0}^{1} d y\left(1-y^{\beta}\right)^{1 / \alpha} .
$$

The integral over $d y$ can be expressed in the terms of the $B$-function? Indeed, substitution $z=y^{\beta}$ yields

$$
\int_{0}^{1} d y\left(1-y^{\beta}\right)^{1 / \alpha}=\frac{1}{\beta} \int_{0}^{1} d z z^{\frac{1}{\beta}-1}(1-z)^{\frac{1}{\alpha}}=\frac{1}{\beta} B\left(\frac{1}{\beta}, \frac{1}{\alpha}+1\right) .
$$

With help of Eqs.(37) and (38) we rewrite Eq.(36) as

$$
2 \pi \hbar\left(n+\frac{1}{2}\right)=\frac{4}{D_{\alpha}^{1 / \alpha} q^{2 / \beta}} E^{\frac{1}{\alpha}+\frac{1}{\beta}} \frac{1}{\beta} B\left(\frac{1}{\beta}, \frac{1}{\alpha}+1\right) .
$$

The above equation gives the value of the energies of stationary states for 1D fractional oscillator,

$$
E_{n}=\left(\frac{\pi \hbar \beta D_{\alpha}^{1 / \alpha} q^{2 / \beta}}{2 B\left(\frac{1}{\beta}, \frac{1}{\alpha}+1\right)}\right)^{\frac{\alpha \beta}{\alpha+\beta}} \cdot\left(n+\frac{1}{2}\right)^{\frac{\alpha \beta}{\alpha+\beta}} .
$$

This new equation generalize the well known energy spectrum of the standard quantum mechanical oscillator (see for example, [22]) and is transformed to it at the special case $\alpha=2, \beta=2$.

As it follows from Eq.(39) at

$$
\frac{\alpha \beta}{\alpha+\beta}=1
$$

the energy spectrum becomes equidistant. When $1<\alpha \leq 2$ and $1<\beta \leq 2$ the condition given by Eq.(40) takes place for $\alpha=2$ and $\beta=2$ only. It means that only standard quantum mechanical oscillator has the equidistant energy spectrum.

\footnotetext{
${ }^{2}$ The $B(a, b)$ function has the familiar integral representation [21]

$B(a, b)=\int_{0}^{1} d u u^{a-1}(1-u)^{b-1}$.
} 


\section{Current density}

By multiplying Eq.(8) from left by $\psi^{*}(\mathbf{r}, t)$ and the conjugate complex of Eq.(8) by $\psi(\mathbf{r}, t)$ and subtracting the two resultant equations we finally obtain

$$
\begin{gathered}
\frac{\partial}{\partial t} \int d^{3} r\left(\psi^{*}(\mathbf{r}, t) \psi(\mathbf{r}, t)\right)= \\
\frac{D_{\alpha}}{i \hbar} \int d^{3} r\left(\psi^{*}(\mathbf{r}, t)\left(-\hbar^{2} \Delta\right)^{\alpha / 2} \psi(\mathbf{r}, t)-\psi(\mathbf{r}, t)\left(-\hbar^{2} \Delta\right)^{\alpha / 2} \psi^{*}(\mathbf{r}, t)\right) .
\end{gathered}
$$

From this integral relationship we are led to the following well known differential equation

$$
\frac{\partial \rho(\mathbf{r}, t)}{\partial t}+\operatorname{div} \mathbf{j}(\mathbf{r}, t)=0
$$

where

$$
\rho(\mathbf{r}, t)=\psi^{*}(\mathbf{r}, t) \psi(\mathbf{r}, t),
$$

is the density of probability and the vector $\mathbf{j}(\mathbf{r}, t)$ can be called by the fractional probability current density vector

$\mathbf{j}(\mathbf{r}, t)=\frac{D_{\alpha} \hbar}{i}\left(\psi^{*}(\mathbf{r}, t)\left(-\hbar^{2} \Delta\right)^{\alpha / 2-1} \nabla \psi(\mathbf{r}, t)-\psi(\mathbf{r}, t)\left(-\hbar^{2} \Delta\right)^{\alpha / 2-1} \nabla \psi^{*}(\mathbf{r}, t)\right)$,

where we use the following notation

$$
\boldsymbol{\nabla}=\frac{\partial}{\partial \mathbf{r}}
$$

Introducing the momentum operator $\widehat{\mathbf{p}}=\frac{\hbar}{i} \boldsymbol{\nabla}$ we can write the vector $\mathbf{j}$ in the form

$$
\mathbf{j}=D_{\alpha}\left(\psi\left(\widehat{\mathbf{p}}^{2}\right)^{\alpha / 2-1} \widehat{\mathbf{p}} \psi^{*}+\psi^{*}\left(\widehat{\mathbf{p}}^{* 2}\right)^{\alpha / 2-1} \widehat{\mathbf{p}}^{*} \psi\right), \quad 1<\alpha \leq 2 .
$$

When $\alpha=2, D_{\alpha}=1 / 2 m$ Eqs.(44), (45) becomes the well known equations of the standard quantum mechanics (see, for example [22]). Thus we conclude that the new Eqs.(44) and (45) are the fractional generalization of 
the well known equations for probability current density vector of standard quantum mechanics.

To this end, we express Eq.(45) in the terms of the velocity operator, which is defined as usual

$$
\widehat{\mathbf{v}}=\frac{d}{d t} \widehat{\mathbf{r}}
$$

where $\widehat{\mathbf{r}}$ is the operator of coordinate. Using the general quantum mechanical rule for differentiation of operator

$$
\frac{d}{d t} \widehat{\mathbf{r}}=\frac{i}{\hbar}\left[H_{\alpha}, \mathbf{r}\right]
$$

we have

$$
\widehat{\mathbf{v}}=\frac{i}{\hbar}\left(H_{\alpha} \mathbf{r}-\mathbf{r} H_{\alpha}\right)
$$

Further, with help of the equation

$$
\mathrm{f}(\widehat{\mathbf{p}}) \mathbf{r}-\mathbf{r f}(\widehat{\mathbf{p}})=-i \hbar \frac{\partial \mathrm{f}}{\partial \mathbf{p}}
$$

which holds for any function $\mathrm{f}(\widehat{\mathbf{p}})$ of the momentum operator, and taking into account Eq.(2) for the Hamiltonian we find the equation for the velocity operator

$$
\widehat{\mathbf{v}}=\alpha D_{\alpha}\left|\widehat{\mathbf{p}}^{2}\right|^{\alpha / 2-1} \widehat{\mathbf{p}}
$$

here $\widehat{\mathbf{p}}$ is the momentum operator. By comparing of Eqs.(45) and (46) we finally conclude that

$$
\mathbf{j}=\frac{1}{\alpha}\left(\psi \widehat{\mathbf{v}} \psi^{*}+\psi^{*} \widehat{\mathbf{v}} \psi\right), \quad 1<\alpha \leq 2 .
$$

To get the probability current density equal 1 (the current when one particle pass through the unit area per unit time) the wave function of a free particle have to be normalized as follows

$$
\psi(\mathbf{r}, t)=\sqrt{\frac{\alpha}{2 \mathrm{v}}} \exp \left\{\frac{i}{\hbar} \mathbf{p r}-\frac{i}{\hbar} E t\right\}, \quad E=D_{\alpha}|\mathbf{p}|^{\alpha}, \quad 1<\alpha \leq 2,
$$


where $\mathrm{v}$ is the particle velocity, $\mathrm{v}=\alpha D_{\alpha} p^{\alpha-1}$. Indeed, by substituting Eq.(48) into Eq.(45) we find

$$
\mathbf{j}=\frac{\mathbf{v}}{\mathrm{V}}, \quad \mathbf{v}=\alpha D_{\alpha}\left|\mathbf{p}^{2}\right|^{\frac{\alpha}{2}-1} \mathbf{p},
$$

that is the vector $\mathbf{j}$ is the unit vector.

Equations (44), (45) and (47) are the fractional generalization of the well known equations for probability current density vector of the standard quantum mechanics [22].

\section{Conclusions}

Fractional generalization of the Schrödinger equation has been studied. We have established the integration by parts formula for the quantum Riesz fractional derivative and used it to prove hermiticity of the fractional Hamilton operator. The parity conservation law for the fractional quantum mechanics has been observed. The time-independent fractional Schrödinger equation has been introduced. As physical applications of the time-independent fractional Schrödinger equation we have found the energy spectrum and equation for the orbits radius for a hydrogen-like atom - fractional "Bohr atom". The energy spectrum of 1D fractional oscillator has been obtained in the semiclassical approximation.

The generalization of the fractional probability current density has been derived and discussed.

The generalized equations (8), (22), (29)-(33), (39), (44), (45) and (47) are transformed into the well known equations of conventional quantum mechanics if we put the Lévy index $\alpha=2$. In other words the fractional quantum mechanics includes the standard quantum mechanics as a particular Gaussian case at $\alpha=2$. Quantum mechanical path integral over the Lévy paths at $\alpha=2$ becomes the well known Feynman path integral and the fractional Schrödinger equation becomes the Schrödinger equation.

The fractional Schrödinger equation provides us with general point of view on the relationship between statistical properties of quantum-mechanical path and structure of the fundamental equations of quantum mechanics. 


\section{References}

[1] R. P. Feynman and A.R. Hibbs, Quantum Mechanics and Path Integrals (McGraw-Hill, New York, 1965).

[2] R.P. Feynman, Statistical Mechanics (Benjamin. Reading, Mass. 1972).

[3] P. Lévy, Théorie de l'Addition des Variables Aléatoires (GauthierVillars, Paris, 1937).

[4] A. Y.Khintchine and P. Lévy, C.R. Acad. Sci. (Paris) 202, 374 (1936).

[5] W. Feller, An Introduction to Probability Theory and its Applications, (John Wiley \& Sons, New York, 1966).

[6] V.M. Zolotarev, One-dimensional Stable Distributions, MM, Vol 65, (AMS 1986).

[7] M. Kac, in the Second Berkeley Symposium on Mathematical Statistics and Probability (University of California Press, Berkeley, Calif., 1951).

[8] N. Laskin, Phys.Lett. A 268, 298 (2000).

[9] N. Laskin, Phys. Rev. E 62, 3135 (2000).

[10] A.I. Saichev and G.M. Zaslavsky, Chaos 7, 753 (1997).

[11] K.B. Oldham and J. Spanier, The Fractional Calculus (Academic Press, New York, 1974).

[12] K.S. Miller and B. Ross, An Introduction to the Fractional Calculus and Fractional Differential Equations, (Wiley, New York, 1993).

[13] S.G. Samko, A.A. Kilbas and O.I. Marichev, Fractional Integrals and Derivatives, Theory and Applications, (Gordon and Breach, Amsterdam, 1993).

[14] I. Podlubny, Fractional Diffrential Equations (Academic Press, New York, 1999).

[15] R. Metzler, J. Klafter, Phys. Rep. 339, 1-77 (2000). 
[16] H. Kleinert, Path Integrals in Quantum Mechanics, Statistics and Polymer Physics (World Scientific, Singapore, 1990).

[17] M. Riesz, Acta Mathematica, 81,1 (1949).

[18] L.D. Landau and E.M. Lifshitz, Mechanics (third edition), Vol.1 of Course of Theoretical Physics (Pergamon Press, Oxford, 1976).

[19] N. Bohr, Phil. Mag. 26, 1, 476, 857 (1913).

[20] N. Bohr, Collected Works, vol.4. ed. J. Rud Nielsen (Amsterdam, NorthHolland, 1977).

[21] A. Erdelyi, Ed., Higer Transcendental Functions (McGraw-Hill, New York, 1955), Vol.3.

[22] L.D. Landau and E.M. Lifshitz, Quantum mechanics (Non-relativistic Theory), Vol.3 of Course of Theoretical Physics (Pergamon Press, London, 1958). 